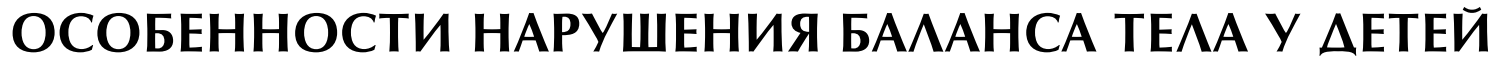 С ОАНОСТОРОННИМ УКОРОЧЕНИЕМ НИЖНЕЙ КОНЕЧНОСТИ
}

\author{
() И.Е. Никитюк ${ }^{1}$ Е.Л. Кононова ${ }^{1}$, Ю.Е. Гаркавенко ${ }^{1,2}$ \\ ${ }^{1}$ ФГБУ «НИДОИ им. Г.И. Турнера» Минздрава России, Санкт-Петербург; \\ ${ }^{2}$ ФГБОУ ВО «СЗГМУ им. И.И. Мечникова» Минздрава России, Санкт-Петербург
}

Поступи^а: 25.02.2019

ОАобрена: 17.07.2019

Принята: 09.09.2019

Обоснование. Проблема одностороннего укорочения нижних конечностей у детей является чрезвычайно актуальной для современной ортопедии. В процессе роста ребенка укорочение пораженного сегмента прогрессирует, что приводит к анатомической асимметрии нижних конечностей и нарастанию дисбаланса нагрузки на них. Инвалидизацию пациента усугубляют вторичные деформации таза и позвоночника. Остаются малоизученными особенности нарушения постурального баланса тела в зависимости от этиологии заболевания врожденного или приобретенного, степень сохранности двигательных стереотипов у детей с односторонним укорочением нижних конечностей.

Цель - изучить постуральную стабильность у детей с односторонним укорочением нижних конечностей и оценить нарушения баланса тела в зависимости от этиологии поражения.

Материалы и методы. Были определены нормативные значения стабилометрических показателей 11 здоровых детей, средний возраст которых составил 11,9 \pm 0,73 года (первая группа), а также параметры статокинезиограмм у 22 пациентов с односторонним укорочением нижней конечности. Из них во вторую группу вошли 11 детей с врожденным укорочением нижней конечности (среднее укорочение $-4,8 \pm 0,80$ см, средний возраст $-11,9 \pm 1,05$ года). Третью группу составляли также 11 детей, но с приобретенным укорочением нижней конечности (среднее укорочение - 4,5 \pm 0,38 см, средний возраст $-12,2 \pm 0,78$ года). Статистическое исследование включало корреляционный анализ.

Результаты. В обеих группах пациентов выявлено значимое снижение стабильности вертикального баланса, проявляющееся выраженными отклонениями от номинальных значений стабилометрических параметров по сравнению со здоровыми детьми: повышенным смещением центра давления, большими значениями площади статокинезиограммы и длины траектории центра давления. При помощи метода стабилометрии определяли состояние адаптивных постуральных механизмов для оценки формирования у пациентов с односторонним укорочением нижней конечности степени адекватности двигательной стратегии в зависимости от этиологии поражения.

Заключение. У пациентов с приобретенным укорочением нижней конечности сформирован адекватный адаптивный двигательный стереотип, система обеспечения постурального баланса стабилизирована в новых условиях. У пациентов с врожденным укорочением нижней конечности была иная стратегия поддержания позной устойчивости, характеризующаяся неоптимальным двигательным стереотипом. Стабилометрическая оценка асимметрии нагрузки на нижние конечности может быть использована для изучения формирования компенсаторных механизмов управления системой локомоции, что важно при планировании реабилитационных мероприятий.

Ключевые слова: укорочение нижней конечности; постуральный контроль; стабилометрия; асимметрия нагрузки на нижние конечности.

\section{CHARACTERISTICS OF BODY BALANCE DISORDER IN CHILDREN WITH UNILATERAL LOWER LIMB SHORTENING}

\author{
(C) I.E. Nikityuk ${ }^{1}$, E.L. Kononova ${ }^{1}$, Yu.E. Garkavenko ${ }^{1,2}$
}

${ }^{1}$ The Turner Scientific Research Institute for Children's Orthopedics, Saint Petersburg, Russia

${ }^{2}$ North-Western State Medical University n.a. I.I. Mechnikov, Saint Petersburg, Russia

For citation: Pediatric Traumatology, Orthopaedics and Reconstructive Surgery. 2019;7(3):45-54 
Background. In modern orthopedics, the problem of unilateral shortening of the lower limbs in children is extremely important. In the process of child growth, there occurs progression of the shortened segment, which leads to anatomical asymmetry of the lower limbs and an increase in the imbalance of the limb load. Secondary deformities of the pelvis and spine aggravate the patient's disability. The features of abnormal postural balance of the body depending on the etiology of the disease, such as congenital or acquired, as well as the degree of preservation of motor stereotypes in children with unilateral shortening of the lower limbs, are still understudied.

Aim. The aims of this work are to study postural stability in children with unilateral shortening of the lower limbs and to assess the disorders of body balance depending on the etiology of the lesion.

Materials and methods. The standard stabilometric values of 11 healthy children (average age, $11.9 \pm 0.73$ years) were determined (group 1), as well as the statokinesiogram parameters in 22 patients with unilateral shortening of the lower limb. The second group included 11 children (average age, $11.9 \pm 1.05$ years) with congenital shortening of the lower limb (average shortening, $4.8 \pm 0.8 \mathrm{~cm}$ ). The third group also consisted of 11 children (average age, $12.2 \pm 0.78$ years), but with acquired shortening of the lower limb (average shortening, $4.5 \pm 0.38 \mathrm{~cm}$ ). Statistical research included correlation analysis.

Results. A significant decrease in the stability of the vertical balance was observed in both groups of patients, which was demonstrated by pronounced deviations from the nominal values of stabilometric parameters, compared with healthy children: an increased center of pressure displacement, large values of the statokinesiogram area, and the length of the pressure displacement path. It was possible to determine the state of adaptive postural mechanisms for assessing the formation of the degree of adequacy of the motor strategy in patients with unilateral shortening of the lower limb, depending on the etiology of the lesion, owing to the method of stabilometry.

Conclusion. An appropriate adaptive motor stereotype has been formed in patients with acquired shortening of the lower limb; in the new conditions, the system for ensuring postural balance is stabilized. There is a different strategy for maintaining posture stability characterized by a nonoptimal motor stereotype in patients with congenital shortening of the lower limb. The stabilometric assessment of the asymmetry of the lower limb load is a promising method for studying the formation of compensatory mechanisms for controlling the locomotion system, which is important when planning rehabilitation measures.

Keywords: shortening of the lower limb; postural control; stabilometry; limb load asymmetry.

\section{Обоснование}

Проблема одностороннего укорочения нижних конечностей у детей является чрезвычайно актуальной для современной ортопедии. В процессе роста ребенка, вследствие врожденного или приобретенного поражения метаэпифизарных зон роста трубчатых костей, укорочение пораженного сегмента прогрессирует, что приводит к вторичным деформациям таза и позвоночника и инвалидизации пациента. Причинами патологии часто служит врожденное или приобретенное нарушение функции метаэпифизарных зон роста длинных трубчатых костей нижних конечностей, развившееся на фоне трофических нарушений [1], однако активность метаэпифизарного хряща изменяется и при полном исключении клеточногуморальных воздействий на него [2]. При этом с возрастом у ребенка прогрессирует анатомическая асимметрия нижних конечностей, приводящая к нарастанию дисбаланса нагрузки на них как при стоянии, так и при ходьбе [3], что обусловливает неравномерное распределение механической нагрузки на ростковую пластинку и дальнейшее нарушение ее функции [4].

В то время как исследованию дисбаланса нагрузки на нижние конечности при их одинаковой длине уделено достаточно внимания при различных как физиологических $[5,6]$, так и патологических $[7,8]$ состояниях, адаптивные возможности опорно-двигательной системы при одностороннем укорочении нижней конечности изучены недостаточно [9]. Остаются также неисследованными особенности нарушения постуральной функции в зависимости от этиологии укорочения нижней конечности и степень сохранности двигательных стереотипов, оценка которых крайне важна при планировании ортопедического восстановительного лечения [10]. Изучение особенностей поддержания вертикального баланса тела при асимметрии нижних конечностей позволяет анализировать механизмы нарушения постурального контроля и оценивать возможное его восстановление после хирургического выравнивания длины нижних конечностей [11]. Используемый в этих целях метод стабилометрии важен как для изучения формирования адаптивных реакций организма в управлении мышечной активностью тела, так и для создания новых технических систем управления движениями [12].

Цель работы - изучить постуральную стабильность у детей с односторонним укорочением нижних конечностей и оценить нарушения баланса тела в зависимости от этиологии поражения. 


\section{Материалы и методы}

Дизайн исследования. Выполнено одномоментное ретроспективное исследование.

Условия проведения исследования. Были определены нормативные значения стабилометрических показателей 11 здоровых детей в возрасте от 8 до 16 лет, средний возраст которых составил $11,9 \pm 0,73$ года (первая группа - контрольная).

Проанализированы истории болезни и результаты лучевого обследования основных групп детей. Во вторую группу были включены 11 детей в возрасте от 8 до 16 лет (средний возраст $-11,9 \pm 1,05$ года) с врожденным укорочением нижней конечности на фоне гипофункции метаэпифизарного росткового хряща бедренной и берцовых костей. Степень вовлеченности в патологический процесс ростковых зон пораженных конечностей не оценивали. Средняя разница в длине нижних конечностей у них составила $\Delta l=4,8 \pm 0,80$ см (рис. $1, a)$. В третью группу были включены также 11 пациентов в возрасте от 9 до 16 лет (средний возраст $-12,2 \pm 0,78$ года) с приобретенным укорочением нижней конечности на фоне деструктивных изменений проксимальной метаэпифизарной зоны роста бедренной кости после перенесенного острого гематогенного остеомиелита. Средняя разница в длине нижних конечностей у них составила $\Delta l=4,5 \pm 0,38 \mathrm{~cm}$ за счет укорочения длины бедра (рис. 1,6$)$. У пациентов обеих групп наблюдалась гипотрофия мягких тканей бедра и голени пораженной конечности различной степени выраженности.

Исследованные группы пациентов были однородными по возрастному составу и по величине укорочения пораженной нижней конечности. Кроме того, ключевым критерием включения в каждую группу являлось отсутствие угловой деформации коленного сустава.

Критерии исключения. Пациенты с поражением росткового хряща костей, формирующих коленный сустав, были исключены из выборки в связи с тем, что у большинства из них развивалась угловая деформация коленного сустава различной направленности.

Meтоды. Стабилометрическое исследование проводили с помощью программно-аппаратного комплекса МБН «Биомеханика» (ООО НМФ «МБН») при «европейской» установке стоп ребенка: стопы располагали на платформе при сведении пяток и разведении передних отделов с углом между внутренними краями стоп $30^{\circ}$. Пациенту предлагали занять удобное для него вертикальное положение стоя с опущенными вдоль тела руками, с опорой на обе нижние конечности. В случае

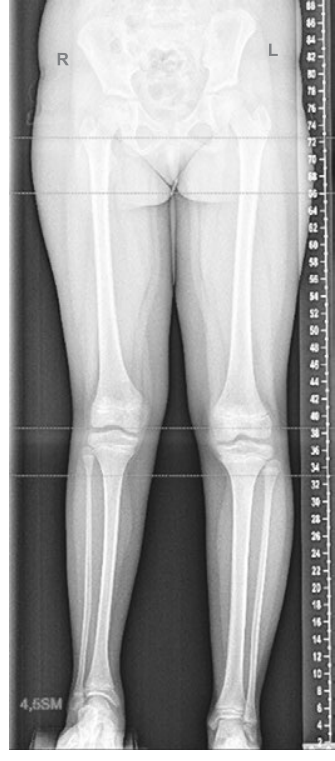

$a$

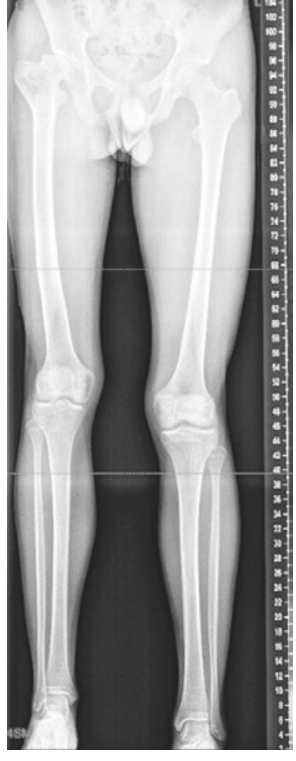

6
Рис. 1. Панорамные рентгенограммы нижних конечностей: $a$ - пациентка К., 10 лет, с врожденным укорочением правой нижней конечности на 4,5 см; 6 - пациент А., 16 лет, с приобретенным укорочением правой нижней конечности на 4,0 см

значительного укорочения нижней конечности ее опорность компенсаторно реализовывалась за счет нагружения только переднего отдела стопы. Исследования проводили по стандартной функциональной пробе с открытыми и закрытыми глазами с регистрацией параметров смещения проекции центра давления (ЦД) тела. Графическим отражением колебаний ЦД являлась стабилограмма, представленная фигурой эллипса разной направленности и с разной выраженностью эксцентриситета (степенью вытянутости эллипса) (рис. 2). Вычисляли девиации ЦД во фронтальной $x$ (мм) и сагиттальной $y$ (мм) плоскостях и рассчитывали соотношения параметров $y$ к $x$, то есть определяли величину отношения длины эллипса статокинезиограммы к его ширине $(y / x)$, для выявления стратегии вертикального баланса у пациентов - фронтального или сагиттального [13].

Определяли следующие параметры: координаты $X$ (мм) и $Y$ (мм) ЦД, среднюю длину траектории, пройденной ЦД ( $L$, мм), площадь $S\left(\right.$ мм$\left.^{2}\right)$. Рассчитывали знак и среднюю величину угла преимущественного направления колебаний относительно сагиттальной плоскости $A l\left({ }^{\circ}\right)$ и его изменение при пробе с открытыми и закрытыми глазами $\Delta A l\left(^{\circ}\right)$.

Статистические методы обработки. Поскольку характер распределения показателей стабилометрии согласно критерию Шапиро - Уилка был определен как непараметрический, для сравнения значений несвязанных выборок использо- 
вали критерий Манна - Уитни. Данные представляли в виде медианы (Ме) и межквартильного интервала (25-75\%). Пороговый уровень статистической значимости принимали при значении критерия $p<0,05$. При анализе связанных выборок использовали критерий Уилкоксона. Для исследования линейной взаимосвязи двух признаков применяли корреляционный анализ с помощью непараметрического коэффициента Спирмена $r_{s}$.

\section{Результаты}

У всех детей с укорочением нижней конечности были выявлены выраженные нарушения постурального баланса, на что указывали данные количественных показателей (табл. 1). Это проявлялось значимым увеличением по сравнению с нормой средних значений $L$ и $S$ колебаний ЦД в обеих группах пациентов, причем значимых различий в указанных показателях между детьми второй и третьей групп не было.

Анализ смещения ЦД во фронтальной плоскости (ось $X$, мм) позволил констатировать асимметричное распределение веса тела на нижние конечности у пациентов обеих групп. При этом пораженная конечность компенсаторно разгружалась, и вес тела перераспределялся в сторону интактной нижней конечности. Средние величины латерального смещения ЦД были значимы для больных с врожденным и приобретенным укорочением, однако не различались между группами пациентов. При этом корреляционная связь величины укорочения нижней конечности $\Delta l$ с координатой $X$ оказалась умеренной в обеих группах как при открытых, так и при закрытых глазах (табл. 2). Такой характер асимметричного распределения веса тела на нижние конечности у пациентов может свидетельствовать о компенсаторном перераспределении

таблица 1

Показатели статокинезиограмм зАоровых детей и пациентов с односторонним укорочением нижней конечности

\begin{tabular}{|c|c|c|c|c|c|}
\hline \multirow{2}{*}{\multicolumn{2}{|c|}{ Параметры }} & \multicolumn{3}{|c|}{ Группы обследованных детей } & \multirow{3}{*}{$\begin{array}{c}\text { Критерий } \\
\text { Манна - Уитни, } \\
p \text {-value } \\
\\
p_{1-2}<0,0001 \\
p_{1-3}=0,0001 \\
p_{2-3}=1,0\end{array}$} \\
\hline & & \multirow{2}{*}{$\begin{array}{c}\text { здоровые }(1) \\
\operatorname{Me}\left(Q_{1}-Q_{2}\right) \\
n=11 \\
0,3 \\
(0,1-0,4)\end{array}$} & \multirow{2}{*}{$\begin{array}{c}\text { с врожденным } \\
\text { укорочением НК (2) } \\
\text { Ме }\left(Q_{1}-Q_{2}\right) \\
n=11 \\
21,9 \\
(2,2-26,0)\end{array}$} & \multirow{2}{*}{$\begin{array}{c}\text { с приобретенным } \\
\text { укорочением НК (3) } \\
\text { Мe }\left(Q_{1}-Q_{2}\right) \\
n=11 \\
11,8 \\
(7,6-27,7)\end{array}$} & \\
\hline$X$, мм & ОГ & & & & \\
\hline & $3 \Gamma$ & $\begin{array}{c}0,3 \\
(0,1-0,4)\end{array}$ & $\begin{array}{c}21,6 \\
(3,3-25,2)\end{array}$ & $\begin{array}{c}11,4 \\
(4,8-19,1)\end{array}$ & $\begin{array}{l}p_{1-2}=0,0007 \\
p_{1-3}<0,0001 \\
p_{2-3}=0,646\end{array}$ \\
\hline \multirow[t]{2}{*}{ Y, мм } & ОГ & $\begin{array}{c}3,7 \\
(2,4-5,2)\end{array}$ & $\begin{array}{c}24,2 \\
(-3,9-37,8)\end{array}$ & $\begin{array}{c}32,8 \\
(28,4-49,4)\end{array}$ & $\begin{array}{l}p_{1-2}=0,293 \\
p_{1-3}<0,0001 \\
p_{2-3}=0,057\end{array}$ \\
\hline & $3 \Gamma$ & $\begin{array}{c}7,7 \\
(4,4-9,5)\end{array}$ & $\begin{array}{c}25,6 \\
(3,3-38,2)\end{array}$ & $\begin{array}{c}37,5 \\
(30,7-51,8)\end{array}$ & $\begin{array}{l}p_{1-2}=0,088 \\
p_{1-3}<0,0001 \\
p_{2-3}=0,03\end{array}$ \\
\hline \multirow[t]{2}{*}{$L, \mathrm{MM}$} & ОГ & $\begin{array}{c}637 \\
(532-705)\end{array}$ & $\begin{array}{c}835 \\
(723-1152)\end{array}$ & $\begin{array}{c}986 \\
(811-1035)\end{array}$ & $\begin{array}{l}p_{1-2}=0,004 \\
p_{1-3}=0,0005 \\
p_{2-3}=0,646\end{array}$ \\
\hline & $3 \Gamma$ & $\begin{array}{c}766 \\
(650-911)\end{array}$ & $\begin{array}{c}1206 \\
(902-1430)\end{array}$ & $\begin{array}{c}1041 \\
(848-1296)\end{array}$ & $\begin{array}{l}p_{1-2}=0,003 \\
p_{1-3}=0,007 \\
p_{2-3}=0,694\end{array}$ \\
\hline \multirow[t]{2}{*}{$S, \mathrm{Mм}^{2}$} & ОГ & $\begin{array}{c}366 \\
(344-621)\end{array}$ & $\begin{array}{c}529 \\
(365-1109)\end{array}$ & $\begin{array}{c}880 \\
(570-1343)\end{array}$ & $\begin{array}{l}p_{1-2}=0,066 \\
p_{1-3}=0,007 \\
p_{2-3}=0,115\end{array}$ \\
\hline & $3 \Gamma$ & $\begin{array}{c}698 \\
(386-806)\end{array}$ & $\begin{array}{c}861 \\
(598-1035)\end{array}$ & $\begin{array}{c}1184 \\
(419-1571)\end{array}$ & $\begin{array}{l}p_{1-2}=0,056 \\
p_{1-3}=0,087 \\
p_{2-3}=0,599\end{array}$ \\
\hline \multicolumn{2}{|c|}{$y / x$} & $\begin{array}{c}1,39 \\
(1,23-1,67)\end{array}$ & $\begin{array}{c}1,18 \\
(0,95-1,40)\end{array}$ & $\begin{array}{c}1,13 \\
(0,86-1,51)\end{array}$ & $\begin{array}{l}p_{1-2}=0,015 \\
p_{1-3}=0,010 \\
p_{2-3}=0,664\end{array}$ \\
\hline
\end{tabular}

Примечание: $p_{1-2 ; 1-3 ; 2-3}-$ уровень значимости различий между группами. ОГ - открытые глаза, ЗГ - закрытые глаза. 
Таблица 2

Корреляционный анализ линейной зависимости смещения центра Аавления по осям $X$ и $Y$ от величины укорочения нижней конечности $\Delta /$ у пациентов

\begin{tabular}{|l|c|c|c|}
\hline \multicolumn{2}{|c|}{ Группы обследованных пациентов } & Коэффициент корреляции Спирмена $r_{s}$ \\
\cline { 3 - 4 } \multicolumn{2}{|c|}{} & $\begin{array}{c}\text { Зависимость } \\
X \sim \Delta l\end{array}$ & $\begin{array}{c}\text { 3ависимость } \\
Y \sim \Delta l\end{array}$ \\
\hline \multirow{2}{*}{$\begin{array}{l}\text { C врожденным укорочением НК } \\
n=11\end{array}$} & ОГ & 0,51 & 0,43 \\
\cline { 2 - 4 } & $3 \Gamma$ & 0,61 & 0,49 \\
\hline $\begin{array}{l}\text { C приобретенным укорочением НК } \\
n=11\end{array}$ & ОГ & 0,58 & 0,22 \\
\cline { 2 - 4 } & ЗГ & 0,51 & 0,45 \\
\hline
\end{tabular}

Примечание. НК - нижние конечности, ОГ - открытые глаза, ЗГ - закрытые глаза.

Таблица 3

Показатели величины угла преимущественного направления колебаний центра Аавления статокинезиограмм зАоровых Аетей и пациентов с односторонним укорочением нижней конечности

\begin{tabular}{|c|c|c|c|c|c|}
\hline \multirow{2}{*}{\multicolumn{2}{|c|}{ Параметры }} & \multicolumn{3}{|c|}{ Группы обследованных детей } & \multirow{4}{*}{$\begin{array}{c}\text { Критерий } \\
\text { Манна - Уитни } \\
p \text {-value } \\
\\
p_{1-2}=0,013 \\
p_{1-3}=0,1 \\
p_{2-3}=0,149\end{array}$} \\
\hline & & здоровые (1) & $\begin{array}{c}\text { с врожденным } \\
\text { укорочением НК (2) }\end{array}$ & $\begin{array}{c}\text { с приобретенным } \\
\text { укорочением НК (3) }\end{array}$ & \\
\hline \multirow[t]{3}{*}{$|A l|,{ }^{\circ}$} & \multirow[t]{2}{*}{ ОГ } & $\begin{array}{c}2,6 \\
(1,1-2,8)\end{array}$ & $\begin{array}{c}5,3 \\
(2,4-7,7)\end{array}$ & $\begin{array}{c}2,7 \\
(1,4-5,3)\end{array}$ & \\
\hline & & $p=0,859$ & $p=0,026$ & $p=0,006$ & \\
\hline & $3 \Gamma$ & $\begin{array}{c}1,4 \\
(0,1-2,9)\end{array}$ & $\begin{array}{c}14,4 \\
(11,5-20,9)\end{array}$ & $\begin{array}{c}9,1 \\
(5,3-20,4)\end{array}$ & $\begin{array}{l}p_{1-2}<0,0001 \\
p_{1-3}<0,0001 \\
p_{2-3}=0,149\end{array}$ \\
\hline \multicolumn{2}{|c|}{$\Delta A l, \stackrel{\circ}{ }$} & $\begin{array}{c}2,5 \\
(0,4-3,0)\end{array}$ & $\begin{array}{c}9,8 \\
(7,2-16,2)\end{array}$ & $\begin{array}{c}7,9 \\
(5,4-19,7)\end{array}$ & $\begin{array}{l}p_{1-2}=0,0005 \\
p_{1-3}=0,007 \\
p_{2-3}=0,599\end{array}$ \\
\hline
\end{tabular}

Примечание: $p_{1-2 ; 1-3 ; 2-3}-$ уровень значимости различий между группами; $p$ - уровень значимости различий в группе при пробах с открытыми и закрытыми глазами (критерий Уилкоксона); | - модуль показателей. НК - нижние конечности, ОГ - открытые глаза, 3Г - закрытые глаза.

статической нагрузки при стоянии в пользу здоровой нижней конечности вследствие снижения опорной функции пораженной нижней конечности.

Анализ смещения ЦД в сагиттальной плоскости (ось $Y$, мм) выявил значимое отклонение кпереди только у пациентов с приобретенным укорочением нижней конечности. Причем средняя величина координаты $Y$ в этой группе значимо отличалась от аналогичного показателя как у здоровых детей, так и у пациентов с врожденным укорочением конечности. В этой же группе пациентов выявлена слабая корреляционная связь между величиной укорочения нижней конечности $\Delta l$ и координатой $Y$ при наличии визуального контроля, переходящая в умеренную связь при проведении тестов с закрытыми глазами. Наоборот, у пациентов с врожденным односторонним укорочением нижних конечностей независимо от участия в контроле позы зрительной афферентации корреляционный анализ выявил устойчивую умеренную зависимость координаты $Y$ от величины укорочения нижней конечности $\Delta l$.
При анализе формы стабилограмм было выявлено значимое снижение по сравнению с нормой средних показателей соотношения $y / x$ в обеих группах, что проявлялось уменьшением вытянутости эллипса в сагиттальной плоскости. Это свидетельствует о наличии в обеих группах пациентов с одинаково выраженной тенденцией равновероятной стратегии колебаний ЦД как в сагиттальной, так и во фронтальной плоскости. Такая стратегия удержания баланса близка к патологической, что может указывать на существенное снижение адаптивных возможностей опорнодвигательной системы у пациентов с асимметрией длины нижних конечностей.

Средний угол направления колебаний центра давления $A l$ у детей с приобретенным укорочением нижней конечности оставался в пределах нормальных значений в условиях сохраненного влияния зрительной афферентации и значимо повышался только при проведении проб с закрытыми глазами (табл. 3). По рассмотренному параметру группа пациентов с врожденным одно- 

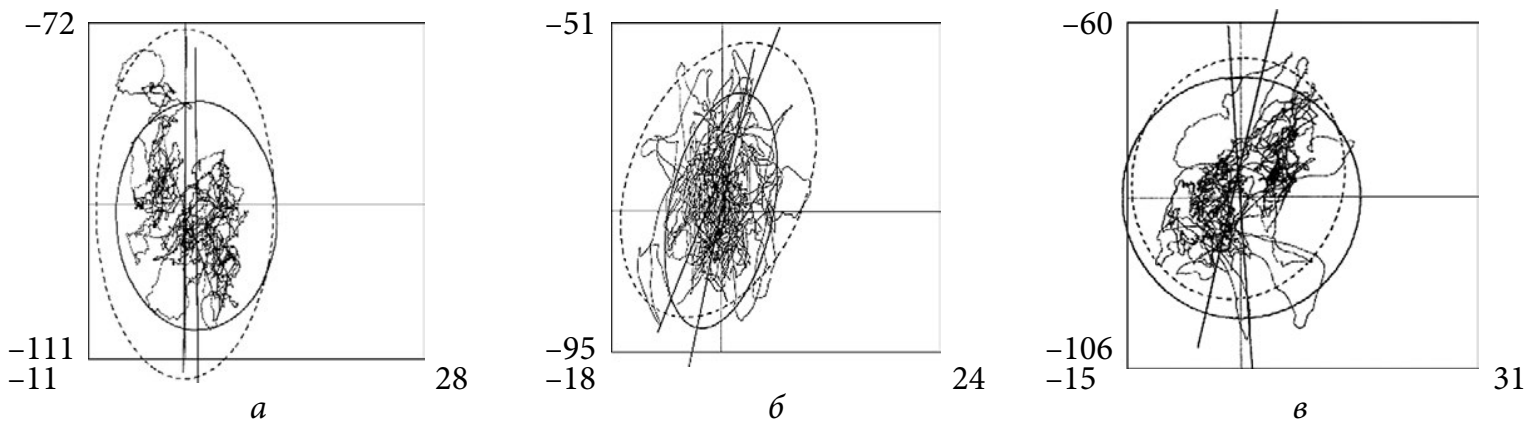

Рис. 2. Знаки угла преимущественного направления колебаний центра давления на статокинезиограммах: $a-$ здоровый ребенок Б., 10 лет, при симметричной нагрузке на нижние конечности; 6 - пациент Н., 12 лет, с врожденным укорочением левой нижней конечности; в - пациент Т., 13 лет, с приобретенным укорочением левой нижней конечности. Длинная ось эллипса - направление колебаний: по часовой стрелке относительно сагиттальной плоскости - положительный угол, против часовой стрелки - отрицательный угол. Сплошная линия при открытых глазах, пунктирная линия - при закрытых глазах

сторонним укорочением нижней конечности имела отличие: средний угол направления колебаний $A l$ значимо превышал таковой у здоровых детей как при зрительном контроле, так и без него.

Величина изменения угла направления колебаний $\Delta A l$ между тестами с открытыми и закрытыми глазами значимо превышала показатель нормы в группе пациентов с врожденным укорочением нижней конечности. В группе пациентов с приобретенным укорочением нижней конечности показатели $\Delta A l$ имели значимые различия с таковыми у здоровых детей только при закрытых глазах. Количественную картину состояния угла направления колебаний $A l$ дополняет качественный анализ.

На статокинезиограммах здоровых детей углы направления колебаний незначительно отклоняются от сагиттальной плоскости независимо от влияния зрительного контроля (рис. 2, a). У детей с врожденным укорочением нижних конечностей направление угла колебаний сохраняется неизменным независимо от участия зрительного контроля и связано со стороной поражения: при левостороннем - положительное значение, при правостороннем - отрицательное (рис. 2, б). А у пациентов с приобретенным укорочением нижних конечностей знак угла направления колебаний ЦД меняется на противоположный в зависимости от состояния зрительной афферентации (рис. 2, в).

Независимо от знака направления колебаний в обеих группах значение модуля угла $A l$ при закрытых глазах было больше, чем при открытых (рис. $3, a, 6$ ), что может свидетельствовать о снижении позной устойчивости при выключении зрительной афферентации.

\section{Обсуждение резумьтатов}

Хорошо известно, что у здоровых людей в обычных условиях вес тела симметрично распределяется между нижними конечностями, которые в равной степени вовлечены в создание момента силы, компенсирующего отклонения тела от положения равновесия. Несимметричное

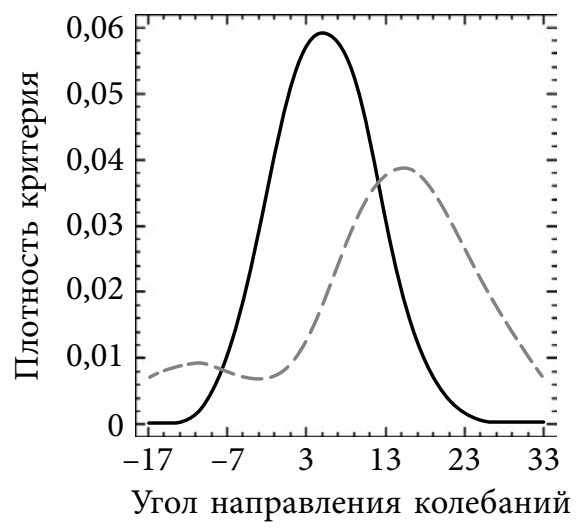

Открытые глаза

$a$

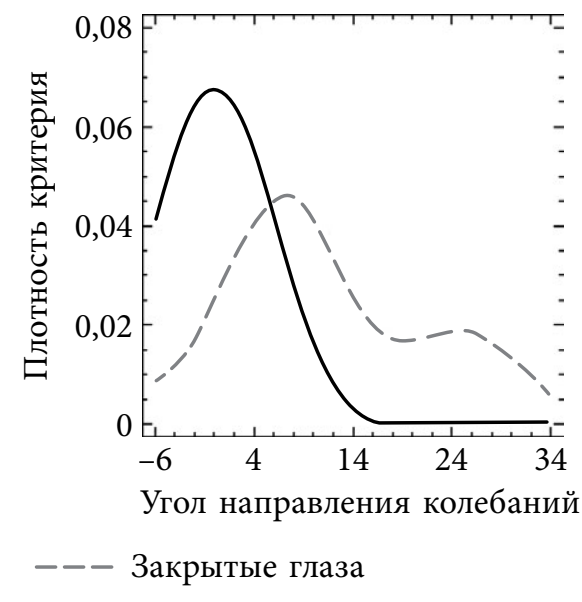

6

Рис. 3. Кривая плотности значений угла преимущественного направления колебаний центра давления на статокинезиограммах пациентов с односторонним укорочением нижних конечностей: $a-c$ врожденным укорочением нижней конечности; б - с приобретенным укорочением нижней конечности 
распределение нагрузки приводит к тому, что более нагруженная нижняя конечность принимает более существенное участие в поддержании ортоградной позы, чем разгруженная [14]. Само нарушение равновесия запускает адаптивные постуральные двигательные реакции [15], а при длительной асимметрии нижних конечностей у пациента формируется патологический двигательный стереотип [16]. В настоящем исследовании у детей с односторонним укорочением нижней конечности врожденного и приобретенного генеза были выявлены выраженные нарушения позной устойчивости. В обеих группах пациентов с асимметрией длины нижних конечностей наблюдалось увеличение по сравнению со здоровыми детьми таких стабилометрических показателей, как $S$ и $L$ статокинезиограмм. Выраженной адаптивной реакцией на одностороннее укорочение нижней конечности явилось повышение нагрузки на интактную конечность вследствие снижения опорной функции пораженной нижней конечности. Дестабилизация вертикальной стойки также наблюдалась и в сагиттальной плоскости, причем в зависимости от генеза укорочения пациенты реализовывали разные стратегии поддержания позной устойчивости. Несмотря на то что у всех пациентов точка опоры стопы пораженной стороны была смещена в передний отдел, у детей с врожденным укорочением нижней конечности не только не происходило значимого смещения ЦД в переднем направлении, но и наблюдалась тенденция к смещению ЦД назад. Напротив, у больных с приобретенным укорочением отмечалось выраженное смещение ЦД вперед, аналогично тому, которое возникает у здоровых людей при искусственном создании легкой неустойчивости вертикальной позы путем произвольного частичного переноса веса тела на одну нижнюю конечность и разгрузки другой [17]. Таким образом, у детей с приобретенным укорочением нижней конечности создается дополнительный статический момент для поддержания вертикальной позы в сагиттальной плоскости, что способствует повышению стабильности баланса тела. Кроме того, в этой группе больных при открытых глазах величина смещения ЦД по оси $Y$ не зависела от укорочения конечности $\Delta l$, что свидетельствует в пользу сохранности физиологического механизма поддержания вертикальной устойчивости. У пациентов с врожденным укорочением нижней конечности смещение ЦД в сагиттальной плоскости хорошо коррелировало с укорочением конечности и не зависело от потока зрительной информации, что указывает на выраженную патологическую установочную реакцию системы управления балансом тела и нарушение стереотипа контроля позы.

Необходимо отметить, что пациенты исследованных групп, несмотря на однородность по возрасту и величине укорочения нижней конечности, различались по уровню поражения ростковых зон костей. Были выявлены различия и в выраженности гипотрофии мышц нижних конечностей. Такие особенности анатомии необходимо учитывать при интерпретации баланса пациентов. В группе детей с врожденным укорочением нижней конечности чаще были вовлечены в патологический процесс ростковые зоны костей, которые формируют коленный сустав, отвечающий за сагиттальный баланс тела. У пациентов с поражением проксимальной метаэпифизарной зоны роста бедренной кости возможно было бы ожидать нарушения вертикального баланса с патологической направленностью колебаний ЦД во фронтальной плоскости. Однако необходимо учитывать, что у детей с хронической разновысокостью нижних конечностей, независимо от этиологии, развиваются компенсаторные изменения в кинематических цепях опорно-двигательной системы. С одной стороны, это проявляется боковым перекосом таза и формированием дуги искривления позвоночника [18]. С другой стороны, все звенья опорно-двигательной системы находятся в многообразных взаимодействиях, что в процессе роста ребенка приводит к неуклонным положительным изменениям биодинамики локомоций [19]. Учитывая, что наибольший вклад в регуляцию вертикального баланса человека наряду с мышцами нижних конечностей вносят мышцы таза и позвоночника [20], их сложное взаимодействие у пациентов с разновысокостью нижних конечностей различного генеза, по всей видимости, приводит к усредненному сбалансированному адаптивному ответу системы постурального контроля на аномально измененные биомеханические условия функционирования организма. В пользу этого предположения могут свидетельствовать результаты настоящей работы по оценке стратегии баланса (фронтального или сагиттального) у обследованных детей: средний показатель соотношений $y / x$ свидетельствует о сагиттальной стратегии баланса у пациентов обеих групп, выраженность которого хотя и была снижена по сравнению с нормой, однако не различалась между двумя группами.

Произвольные значения знака угла $A l$ направленности колебаний ЦД у пациентов с приобретенным укорочением нижней конечности соответствуют принципу распределения направленности осей статокинезиограммы, характерного для здоровых детей. Именно поэтому согласно ре- 
зультатам настоящего исследования частично разгруженная нижняя конечность активно участвует в позном контроле у пациентов с приобретенным укорочением, что может служить признаком формирования у них близкого к физиологичному адаптивного двигательного стереотипа. Это означает, что у таких пациентов система обеспечения постурального баланса тела адекватно стабилизировалась в новых условиях.

$\mathrm{У}$ детей с врожденным укорочением нижней конечности при выключении зрительного контроля знак угла $A l$ направленности колебаний ЦД сохранялся неизменным. Таким образом, уменьшение потока афферентной информации в связи со зрительной депривацией не сопровождалось сенсорной коррекцией движений центра давления за счет проприоцепторов. Такая двигательная стратегия, лишенная преимуществ визуальной системы регуляции, проявляется дестабилизацией системы постурального баланса, так как при отсутствии зрительного контроля механизм поддержания вертикальной устойчивости не обеспечивает в полной мере коррекции позы тела ребенка в пространственных плоскостях. Указанная программа двигательной активности, отвечающая за эффективность позного контроля, считается неоптимальной или патологической [21]. Это позволяет предполагать у пациентов с врожденным укорочением нижней конечности формирование неоптимального двигательного стереотипа.

Следует подчеркнуть, что в настоящем исследовании принимали участие дети только с неосложненной формой асимметрии нижних конечностей - у них была сохранена ось укороченной конечности, поэтому данные, приведенные в настоящем исследовании, не отражают все аспекты проблемы, требуют осторожной интерпретации и не могут распространяться на пациентов с разновысокостью нижних конечностей в сочетании с их деформациями.

\section{Заключение}

Метод стабилометрии позволяет определять состояние адаптивных постуральных механизмов для оценки формирования у пациентов с односторонним укорочением нижней конечности степени адекватности двигательного стереотипа в зависимости от этиологии поражения. Установлено, что у больных с приобретенным укорочением нижней конечности формируется адекватный адаптивный двигательный стереотип, близкий к физиологическому. У больных с врожденным укорочением нижней конечности наблюдается иная стратегия поддержания позной устойчивости, характеризу- ющаяся формированием неоптимального двигательного стереотипа в виде отсутствия компенсаторного смещения ЦД кпереди по сагиттальной составляющей, а также неизменности угла преимущественного направления колебаний, не зависящего от влияния зрительной афферентации. Патологическую постуральную реакцию у таких пациентов можно рассматривать как критерий снижения адаптивных возможностей позной устойчивости. Предложенная методология диагностики двигательных расстройств перспективна для оценки эффективности формирования новой моторной программы у пациентов после оперативного удлинения нижней конечности методом чрескостного дистракционного остеосинтеза.

\section{Аополнительная информация}

Источник финансирования. Работа проведена в рамках выполнения Государственного задания Министерства здравоохранения Российской Федерации, НИР № АААА-А18-118122690156-8.

Конфликт интересов. Авторы декларируют отсутствие явных и потенциальных конфликтов интересов, связанных с публикацией настоящей статьи.

Этическая экспертиза. Исследование выполнено в соответствии с этическими стандартами Хельсинкской декларации Всемирной медицинской ассоциации с поправками Минздрава России, одобрено этическим комитетом ФГБУ «НИДОИ им. Г.И. Турнера» Минздрава России (протокол № 4 от 27.11.2018). Пациенты (их представители) подписали добровольное информированное согласие на обработку и публикацию персональных данных.

Благодарность. Авторы выражают свою благодарность Алене Николаевне Мельченко, руководителю отдела по реализации международных проектов и внешним связям ФГБУ НИДОИ им. Г.И. Турнера Минздрава России за оказанную помощь в переводе на английский язык резюмирующей части публикации.

\section{Вклад авторов}

И.Е. Никитюк - разработка методологии исследования, обработка данных, написание всех разделов статьи, сбор литературных данных и их обработка.

Е.Л. Кононова - проведение исследования и обработка данных.

Ю.Е. Гаркавенко - редактирование текста статьи.

\section{ヘитература}

1. Nguyen JC, Markhardt BK, Merrow AC, Dwek JR. Imaging of pediatric growth plate disturbances. Radiographics. 2017;37(6):1791-1812. https://doi. org/10.1148/rg.2017170029. 
2. Никитюк И.Е., Попов И.В., Полянский В.А., Дудкин В.И. Структура хряща метаэпифизарных пластинок роста в условиях полной изоляции от клеточно-гуморальных воздействий при пересадке в мягкие ткани (экспериментальное исследование) // Морфология. - 2007. - Т. 131. - № 3. C. 45-49. [Nikityuk IE, Popov IV, Polyanskiy VA, Dudkin VI. Structure of metaepiphyseal growth plate cartilage as influenced by total isolation from cellular and humoral influences after its transplantation into soft tissues (an experimental study). Morfologiia. 2007;131(3):45-49. (In Russ.)]

3. Щуров В.А., Новиков К.И., Мурадисинов С.О. Влияние разновысокости нижних конечностей на биомеханические параметры ходьбы // Российский журнал биомеханики. - 2011. - Т. 15. - № 4. - С. 102-107. [Shchurov VA, Novikov KI, Muradisinov SO. Vliyanie raznovysokosti nizhnikh konechnostey na biomekhanicheskie parametry khod'by. Rossiyskiy zhurnal biomekhaniki. 2011;15(4):102-107. (In Russ.)]

4. Guevara JM, Moncayo MA, Vaca-Gonzalez JJ, et al. Growth plate stress distribution implications during bone development: a simple framework computational approach. Comput Methods Programs Biomed. 2015;118(1):59-68. https://doi.org/10.1016/j. cmpb.2014.10.007.

5. Kilby MC, Newell KM. Intra- and inter-foot coordination in quiet standing: footwear and posture effects. Gait Posture. 2012;35(3):511-516. https://doi. org/10.1016/j.gaitpost.2011.11.018.

6. Казенников О.В., Киреева Т.Б., Шлыков В.Ю. Поддержание вертикальной позы человека при асимметричной нагрузке на ноги и односторонней фиксации коленного сустава // Физиология человека. - 2018. - Т. 44. - № 1. - C. 49-55. [Kazennikov OV, Kireeva TB, Shlykov VY. Maintenance of human vertical posture upon asymmetric leg loading and fixation of the knee joint of one leg. Fiziol Cheloveka. 2018;44(1):49-55. (In Russ.)]. https://doi.org/10.7868/ S013116461801-0014.

7. Aruin AS, Kanekar N. Effect of a textured insole on balance and gait symmetry. Exp Brain Res. 2013;231(2):201-208. https://doi.org/10.1007/s00221013-3685-Z.

8. Boonstra TA, Schouten AC, van Vugt JP, et al. Parkinson's disease patients compensate for balance control asymmetry. J Neurophysiol. 2014;112(12):3227-3239. https://doi.org/10.1152/jn.00813.2013.

9. Никитюк И.Е., Гаркавенко Ю.Е., Кононова Е.Л. Особенности опорной функции нижних конечностей у детей с последствиями поражения проксимального отдела бедра острым гематогенным остеомиелитом // Ортопедия, травматология и восстановительная хирургия детского возраста. - 2018. - T. 6. - № 1. - C. 14-22. [Nikityuk IE, Garkavenko YE, Kononova EL. Special aspects of the support function of lower limbs in children with the consequences of unilateral lesion of the proximal femur with acute hematogenous osteomyelitis. Pediatric traumatology, orthopaedics and reconstructive surgery. 2018;6(1):14-22. (In Russ.)]. https://doi.org/10.17816/ PTORS6114-22.
10. Kumar SN, Omar B, Joseph LH, et al. Evaluation of limb load asymmetry using two new mathematical models. Glob J Health Sci. 2014;7(2):1-7. https://doi. org/10.5539/gjhs.v7n2p1.

11. Шеин А.П., Сайфутдинов М.С., Криворучко Г.А. Локальные и системные реакции сенсомоторных структур на удлинение и ишемию конечностей. - Курган: ДАММИ, 2006. - 284 с. [Shein AP, Sayfutdinov MS, Krivoruchko GA. Lokal'nye i sistemnye reaktsii sensomotornykh struktur na udlinenie i ishemiyu konechnostey. Kurgan: DAMMI; 2006. 284 p. (In Russ.)]

12. Грибанов А.В., Шерстенникова А.К. Физиологические механизмы регуляции постурального баланса человека (обзор) // Журнал медико-биологических исследований. - 2013. - № 4. - С. 20-29. [Gribanov AV, Sherstennikova AK. Physiological mechanisms of human postural balance regulation (review). Medical and biological sciences. 2013;(4):20-29. (In Russ.)]

13. Скворцов Д.В. Диагностика двигательной патологии инструментальными методами: анализ походки, стабилометрия. - M.: Т.M. Андреева, 2007. - 640 с. (Skvortsov DV. Diagnostika dvigatel'noy patologii instrumental'nymi metodami: analiz pokhodki, stabilometriya. Moscow: T.M. Andreeva; 2007. 640 p. (In Russ.))

14. Казенников О.В., Киреева Т.Б., Шлыков В.Ю. Влияние структуры опорной поверхности под стопой на поддержание вертикальной позы при разном распределении нагрузки между ногами // Физиология человека. - 2016. - Т. 42. - № 4. - С. 61-68. [Kazennikov OV, Kireeva TB, Shlykov VY. Influence of the structure of the support surface under the sole on vertical posture during standing with different body weight distributions between legs. Fiziol Cheloveka. 2016;42(4):61-68. (In Russ.)]. https://doi.org/10.7868/ S0131164616040044.

15. Wilson EL, Madigan ML, Davidson BS, Nussbaum MA. Postural strategy changes with fatigue of the lumbar extensor muscles. Gait Posture. 2006;23(3):348-354. https://doi.org/10.1016/j.gaitpost.2005.04.005.

16. Айдаров В.И., Скворцов А.П. Формирование правильного двигательного стереотипа ходьбы у пациентов с дефицитом локомоторных функций нижних конечностей // Практическая медицина. - 2013. - T. 2. - № 1-2. - C. 12-13. [Aydarov VI, Skvortsov AP. Formation of correct movement pattern of walking in patients with a deficiency of locomotor functions of the lower limbs. Prakticheskaya meditsina. 2013;2(1-2):12-13. (In Russ.)]

17. Казенников О.В., Киреева Т.Б., Шлыков В.Ю. Особенности поддержания вертикальной позы при неравномерной нагрузке на ноги // Физиология человека. - 2013. - Т. 39. - № 4. - С. 65-73. [Kazennikov OV, Kireeva TB, Shlykov VY. Characteristics of the maintenance of the vertical posture during standing with an asymmetrical load on the legs. Fiziol Cheloveka. 2013;39(4):65-73. (In Russ.)]. https://doi. org/10.7868/S0131164613030119.

18. Щеколова Н.Б., Лихачева Л.В. Динамика биомеханических и электромиографических изменений при 
консервативном лечении детей с идиопатическим укорочением нижних конечностей // Пермский медицинский журнал. - 2013. - Т. 30. - № 1. C. 73-78. [Schekolova NB, Likhacheva LV. Dynamics of biomechanical and electromyographic changes in conservative treatment of children with idiopathic low limb shortening. Permskii meditsinskii zhurnal. 2013;30(1):73-78. (In Russ.)]

19. Бальсевич В.К. Очерки по возрастной кинезиологии человека. - М.: Советский спорт, 2009. - 220 с. [Bal'sevich VK. Ocherki po vozrastnoy kineziologii cheloveka. Moscow: Sovetskiy sport; 2009. 220 p. (In Russ.)]

20. Абдуразаков У.А., Абдуразаков А.У., Юлдашев А.Ж. Асимметрия тела человека: внешние проявления и взаимосвязь с заболеваниями опорно-двига- тельной системы // Вестник Алматинского государственного института усовершенствования врачей. - 2016. - № 4. - С. 28-31. [Abdurazakov UA, Abdurazakov AU, Yuldashev AZh. Asymmetry of a body of the person, external manifestations and interrelation with diseases of musculoskeletal system. Vestnik Almatinskogo gosudarstvennogo instituta usovershenstvovaniya vrachey. 2016;(4):28-31. (In Russ.)]

21. Михайлов В.П., Кузьмичев А.А., Ковтун М.В., Полосухин А.Д. Миофасциальные болевые синдромы и возможности их немедикаментозной коррекции // Хирургия позвоночника. - 2010. - № 4. C. 55-60. [Mikhaylov VP, Kuz'michev AA, Kovtun MV, Polosukhin AD. The role of myofascial structures in the development of pain syndromes and their drug-free correction. Spine surgery. 2010;(4):55-60. (In Russ.)]
Игорь Евгеньевич Никитюк* - канд. мед. наук, ведущий научный сотрудник лаборатории физиологических и биомеханических исследований ФГБУ «НИДОИ им. Г.И. Турнера» Минздрава России. https://orcid. org/0000-0001-5546-2729. E-mail: femtotech@mail.ru.

Елизавета Леонидовна Кононова - канд. мед. наук, старший научный сотрудник лаборатории физиологических и биомеханических исследований ФГБУ «НИДОИ им. Г.И. Турнера» Минздрава России. https:// orcid.org/0000-0001-7624-013X. E-mail: Yelisaveta@ yandex.ru.

Юрий Евгеньевич Гаркавенко - д-р мед. наук, профессор кафедры детской травматологии и ортопедии ФГБОУ ВО «СЗГМУ им. И.И. Мечникова» Минздрава России, ведущий научный сотрудник отделения костной патологии ФГБУ «НИДОИ им. Г.И. Турнера» Минздрава России. https://orcid.org/0000-0001-96618718. E-mail: yurijgarkavenko@mail.ru.
Igor E. Nikityuk ${ }^{*}-\mathrm{MD}, \mathrm{PhD}$, Leading Researcher of the Laboratory of Physiological and Biomechanical Research. The Turner Scientific Research Institute for Children's Orthopedics, Saint Petersburg, Russia. https://orcid. org/0000-0001-5546-2729. E-mail: femtotech@mail.ru.

Elizaveta L. Kononova - MD, $\mathrm{PhD}$, Senior Research Associate of the Laboratory of Physiological and Biomechanical Research. The Turner Scientific Research Institute for Children's Orthopedics, Saint Petersburg, Russia. https://orcid.org/0000-0001-7624-013X. E-mail: Yelisaveta@yandex.ru.

Yuriy E. Garkavenko - MD, PhD, D.Sc., Professor of the Chair of Pediatric Traumatology and Orthopedics. NorthWestern State Medical University n.a. I.I. Mechnikov; Leading Research Associate of the Department of Bone Pathology of The Turner Scientific Research Institute for Children's Orthopedics, Saint Petersburg, Russia. https:// orcid.org/0000-0001-9661-8718. E-mail: yurijgarkavenko@ mail.ru. 